\title{
Analysis of $\boldsymbol{B} \rightarrow \boldsymbol{K} *_{\boldsymbol{J}}(\rightarrow \boldsymbol{K} \pi) \boldsymbol{\mu}^{+} \boldsymbol{\mu}^{-}$in the higher kaon resonance region
}

\author{
Cai-Dian $\mathrm{Lü}^{1}$ and Wei Wang ${ }^{2, *}$ \\ ${ }^{1}$ Institute of High Energy Physics, P.O. Box 918(4), Beijing 100049, People's Republic of China \\ ${ }^{2}$ Deutsches Elektronen-Synchrotron DESY, Hamburg 22607, Germany
}

(Received 15 November 2011; published 8 February 2012)

\begin{abstract}
We study the resonant contributions in the process of $\bar{B}^{0} \rightarrow K^{-} \pi^{+} \mu^{+} \mu^{-}$with the $K^{-} \pi^{+}$invariant mass square $m_{K \pi}^{2} \in[1,5] \mathrm{GeV}^{2}$. Width effects of the involved strange mesons, $K^{*}(1410), K_{0}^{*}(1430)$, $K_{2}^{*}(1430), K^{*}(1680), K_{3}^{*}(1780)$ and $K_{4}^{*}(2045)$, are incorporated. In terms of helicity amplitudes, we derive a compact form for the full angular distributions, through which the branching ratios, forward-backward asymmetries and polarizations are attained. We propose that the uncertainties in the $B \rightarrow K_{J}^{*}$ form factors can be pinned down by the measurements of a set of SU(3)-related processes. Using results from the large energy limit, we derive the dependence of branching fractions on the $m_{K \pi}$, and find that the $K_{2}^{*}$ resonance has a clear signature, in particular, in the transverse polarizations.
\end{abstract}

DOI: $10.1103 /$ PhysRevD.85.034014

PACS numbers: 13.20.He, 12.39.St, 14.40.Be

\section{INTRODUCTION}

It is anticipated that the LHC is able to answer some of the fundamental questions in particle physics. One of great interests is in determining whether the new degrees of freedom are relevant for the phenomena at the TeV scale. On the one hand, many new particles have signatures different from the standard model (SM) particles, and measurements of their production and decays at the LHC may provide definitive evidence on their existence. On the other hand, low energy processes may also be influenced by them. Rare $B$ decays, with tiny decay probabilities in the SM, are highly sensitive to the new degrees of freedom and thus can be exploited as indirect searches of these unknown effects. In particular, $b \rightarrow s l^{+} l^{-}$especially $B \rightarrow$ $K^{*}(\rightarrow K \pi) l^{+} l^{-}$provide a wealth of information on the weak interactions, in terms of a number of observables ranging from the decay probabilities, forward-backward asymmetries (FBAs), polarizations to a full angular analysis. The small branching fraction, of order $10^{-6}$ for $B \rightarrow$ $K^{*} l^{+} l^{-}$, is compensated by the high luminosity at the $B$ factories and hadron colliders [1-3]. It is anticipated that the measurements by the LHCb detector will allow to probe the short-distance physics at an unprecedented level and will provide good sensitivity to discriminate between the SM and different models of new physics. For instance, results by the LHCb based on the data with the integrated luminosity $0.3 \mathrm{fb}^{-1}$ [4] are in good agreement with the theory predictions [5], which has placed a stringent constraint on new physics (NP) models.

In our previous work [6], we have explored the $B \rightarrow$ $K_{2}^{*} l^{+} l^{-}$decay mode in the SM and two specific NP scenarios using the $B \rightarrow K_{2}^{*}$ form factors calculated in Ref. [7]. We provided a comprehensive analysis of the

*wei.wang@desy.de branching ratio, FBAs, transversity amplitudes, and full angular distributions. It is pointed out that the $B \rightarrow K_{2}^{*} l^{+} l^{-}$ decay has several advantages in different aspects and is complementary to the commonly-studied mode $B \rightarrow$ $K^{*} l^{+} l^{-}$. The process $B \rightarrow K_{2}^{*} l^{+} l^{-}$has also received considerable attention in the SM and several variants of it in Refs. [8-13]. On the experimental side, however, its usefulness is challenged by the "pollution" from several other strange resonances in this mass region namely $K^{*}(1410)$, $K_{0}^{*}(1430), K^{*}(1680), K_{3}^{*}(1780)$ and $K_{4}^{*}(2045)$ [14]. The different contributions can be separated by a partial wave analysis when a large amount of data is available, but it is necessary to explore the interference effects in physical quantities, such as the branching ratios to have a benchmark for the possible measurement in the first running of LHC. The aim of the present work is to achieve this goal. To do so, we will study the $B \rightarrow K_{J}^{*} l^{+} l^{-} \rightarrow K \pi l^{+} l^{-}$with the invariant mass square $m_{K \pi}$ ranging from 1 to $5 \mathrm{GeV}^{2}$. Using the helicity amplitudes technique we will derive a compact form for the angular distributions and a number of other quantities. To reduce the uncertainties in $B \rightarrow K_{J}^{*}$ transition form factors, we will propose to measure a set of useful but SU(3)-related channels. In terms of the results derived from the large energy symmetry, we will show the differential distributions and their dependence on $m_{K \pi}^{2}$. We further point out that for $m_{K \pi}^{2} \simeq 2 \mathrm{GeV}^{2}$, the $K_{2}^{*}$ dominates especially in the transverse polarization while at $m_{K \pi}^{2} \simeq$ $3 \mathrm{GeV}^{2}$, the $B \rightarrow K^{*}(1680)$ contribution is the largest.

The rest of the paper is organized as follows. In Sec. II, we give the theoretical framework including the effective Hamiltonian and the hadronic form factors. Section III is devoted to the derivation of the differential decay distributions and the integrated quantities. Section IV is devoted to the numerical predictions in the SM. We conclude in the last section. The Appendix contains our derivation of the angular distributions. 


\section{THEORETICAL FRAMEWORK}

The decay amplitude for $B \rightarrow K_{J}^{*}(\rightarrow K \pi) l^{+} l^{-}$consists of two separate parts: the short-distance physics and the long-distance physics. The former arises from the degrees of freedom higher than $m_{b}$, and thus can be computed by perturbation theory. The low-energy effect is usually parameterized in terms of heavy-to-light form factors.

The $b \rightarrow s l^{+} l^{-}$effective Hamiltonian

$$
\mathcal{H}_{\mathrm{eff}}=-\frac{G_{F}}{\sqrt{2}} V_{t b} V_{t s}^{*} \sum_{i=1}^{10} C_{i}(\mu) O_{i}(\mu)
$$

involves the four-quark and the magnetic penguin operators $O_{i}$ and their explicit forms can be found in Ref. [15]. Here $C_{i}(\mu)$ are the Wilson coefficients for these local operators $O_{i}$, and we use the leading logarithmic values
TABLE I. Wilson coefficients $C_{i}\left(m_{b}\right)$ in the leading logarithmic approximation, with $m_{W}=80.4 \mathrm{GeV}, \mu=m_{b \text {,pole }}$ [15].

\begin{tabular}{ccccccccc}
\hline \hline$C_{1}$ & $C_{2}$ & $C_{3}$ & $C_{4}$ & $C_{5}$ & $C_{6}$ & $C_{7}^{\text {eff }}$ & $C_{9}$ & $C_{10}$ \\
\hline 1.107 & -0.248 & -0.011 & -0.026 & -0.007 & -0.031 & -0.313 & 4.344 & -4.669 \\
\hline \hline
\end{tabular}

[15], which are listed in Table I, $G_{F}$ is the Fermi constant, $V_{t b}=0.999176$ and $V_{t s}=-0.03972[14]$ are the CKM matrix elements. The double Cabibbo suppressed terms, proportional to $V_{u b} V_{u s}^{*}$, have been omitted. $m_{b}=$ $4.67_{-0.06}^{+0.18} \mathrm{GeV}$ and $m_{s}=0.101_{-0.021}^{+0.029} \mathrm{GeV}$ are the $b$ and $s$ quark masses [14].

With the neglect of QCD corrections, only the operators $O_{7 \gamma}, O_{9}$ and $O_{10}$ contribute to the decay amplitudes

$$
\begin{aligned}
i \mathcal{M}\left(b \rightarrow s l^{+} l^{-}\right)= & \frac{i G_{F}}{\sqrt{2}} \frac{\alpha_{\mathrm{em}}}{\pi} V_{t b} V_{t s}^{*} \times\left(\frac{C_{9}+C_{10}}{4}[\bar{s} b]_{V-A}[\bar{l} l]_{V+A}+\frac{C_{9}-C_{10}}{4}[\bar{s} b]_{V-A}[\bar{l} l]_{V-A}\right. \\
& \left.+C_{7 L} m_{b}\left[\bar{s} i \sigma_{\mu \nu}\left(1+\gamma_{5}\right) b\right] \frac{q^{\mu}}{q^{2}} \times\left[\bar{l} \gamma^{\nu} l\right]+C_{7 R} m_{b}\left[\bar{s} i \sigma_{\mu \nu}\left(1-\gamma_{5}\right) b\right] \frac{q^{\mu}}{q^{2}} \times\left[\bar{l} \gamma^{\nu} l\right]\right),
\end{aligned}
$$

where $C_{7 L}=C_{7}$ and $C_{7 R}=\frac{m_{s}}{m_{b}} C_{7 L}$. On the other hand, the operators $O_{1}-O_{6}$ also contribute starting from the one loop diagrams. The factorizable loop terms can be incorporated into the Wilson coefficients $C_{7}$ and $C_{9}$, and thus it is convenient to define the Wilson coefficients combinations $C_{7}^{\text {eff }}$ and $C_{9}^{\text {eff }}[16]$

$$
\begin{aligned}
C_{7}^{\mathrm{eff}}= & C_{7}-C_{5} / 3-C_{6}, \\
C_{9}^{\mathrm{eff}}\left(q^{2}\right)= & C_{9}(\mu)+h\left(\hat{m}_{c}, \hat{s}\right) C_{0}-\frac{1}{2} h(1, \hat{s})\left(4 C_{3}+4 C_{4}+3 C_{5}+C_{6}\right)-\frac{1}{2} h(0, \hat{s})\left(C_{3}+3 C_{4}\right) \\
& +\frac{2}{9}\left(3 C_{3}+C_{4}+3 C_{5}+C_{6}\right),
\end{aligned}
$$

with $\hat{s}=q^{2} / m_{b}^{2}, C_{0}=C_{1}+3 C_{2}+3 C_{3}+C_{4}+3 C_{5}+C_{6}$, and $\hat{m}_{c}=m_{c} / m_{b}$. The auxiliary functions used above are

$$
\begin{aligned}
& h(z, \hat{s})=-\frac{8}{9} \ln \frac{m_{b}}{\mu}-\frac{8}{9} \ln z+\frac{8}{27}+\frac{4}{9} x-\frac{2}{9}(2+x)|1-x|^{1 / 2} \begin{cases}\ln \left|\frac{\sqrt{1-x}+1}{\sqrt{1-x}-1}\right|-i \pi & \text { for } x \equiv \frac{4 z^{2}}{\hat{s}}<1 \\
2 \arctan \frac{1}{\sqrt{x-1}} & \text { for } x \equiv \frac{4 z^{2}}{\hat{s}}>1\end{cases} \\
& h(0, \hat{s})=-\frac{8}{9} \ln \frac{m_{b}}{\mu}-\frac{4}{9} \ln \hat{s}+\frac{8}{27}+\frac{4}{9} i \pi .
\end{aligned}
$$

In the following, we shall also drop the superscripts for $C_{9}^{\text {eff }}$ and $C_{7}^{\text {eff }}$ for brevity.

Properties of the resonances $K_{J}^{*}$ to be explored in this work are collected in Table II. The $B \rightarrow K_{0}^{*}(1430)$ transition form factors are defined by

$$
\begin{aligned}
\left\langle K_{0}^{*}\left(P_{2}\right)\left|\bar{s} \gamma_{\mu} \gamma_{5} b\right| \bar{B}\left(P_{B}\right)\right\rangle & =-i\left\{\left[P_{\mu}-\frac{m_{B}^{2}-m_{K_{0}^{*}}^{2}}{q^{2}} q_{\mu}\right] F_{1}\left(q^{2}\right)+\frac{m_{B}^{2}-m_{K_{0}^{*}}^{2}}{q^{2}} q_{\mu} F_{0}\left(q^{2}\right)\right\}, \\
\left\langle K_{0}^{*}\left(P_{2}\right)\left|\bar{s} \sigma_{\mu \nu} q^{\nu} \gamma_{5} b\right| \bar{B}\left(P_{B}\right)\right\rangle & =\left[\left(m_{B}^{2}-m_{K_{0}^{*}}^{2}\right) q_{\mu}-q^{2} P_{\mu}\right] \frac{F_{T}\left(q^{2}\right)}{m_{B}+m_{K_{0}^{*}}},
\end{aligned}
$$

while the parametrization of the $B \rightarrow K_{J}^{*}(J \geq 1)$ form factors is as follows [10,17] 


$$
\begin{aligned}
\left\langle K_{J}^{*}\left(P_{2}, \epsilon\right)\left|\bar{s} \gamma^{\mu} b\right| \bar{B}\left(P_{B}\right)\right\rangle= & -\frac{2 V\left(q^{2}\right)}{m_{B}+m_{K_{J}^{*}}} \epsilon^{\mu \nu \rho \sigma} \epsilon_{J \nu}^{*} P_{B \rho} P_{2 \sigma}, \\
\left\langle K_{J}^{*}\left(P_{2}, \epsilon\right)\left|\bar{s} \gamma^{\mu} \gamma_{5} b\right| \bar{B}\left(P_{B}\right)\right\rangle= & 2 i m_{K_{J}^{*}} A_{0}\left(q^{2}\right) \frac{\epsilon_{J}^{*} \cdot q}{q^{2}} q^{\mu}+i\left(m_{B}+m_{K_{J}^{*}}\right) A_{1}\left(q^{2}\right)\left[\epsilon_{J \mu}^{*}-\frac{\epsilon_{J}^{*} \cdot q}{q^{2}} q^{\mu}\right] \\
& -i A_{2}\left(q^{2}\right) \frac{\epsilon_{J}^{*} \cdot q}{m_{B}+m_{K_{J}^{*}}}\left[P^{\mu}-\frac{m_{B}^{2}-m_{K_{J}^{*}}^{2}}{q^{2}} q^{\mu}\right], \\
\left\langle K_{J}^{*}\left(P_{2}, \epsilon\right)\left|\bar{s} \sigma^{\mu \nu} q_{\nu} b\right| \bar{B}\left(P_{B}\right)\right\rangle= & -2 i T_{1}\left(q^{2}\right) \epsilon^{\mu \nu \rho \sigma} \epsilon_{J \nu}^{*} P_{B \rho} P_{2 \sigma}, \\
\left\langle K_{J}^{*}\left(P_{2}, \epsilon\right)\left|\bar{s} \sigma^{\mu \nu} \gamma_{5} q_{\nu} b\right| \bar{B}\left(P_{B}\right)\right\rangle= & T_{2}\left(q^{2}\right)\left[\left(m_{B}^{2}-m_{K_{J}^{*}}^{2} \epsilon_{J \mu}^{*}-\epsilon_{J}^{*} \cdot q P^{\mu}\right]+T_{3}\left(q^{2}\right) \epsilon_{J}^{*} \cdot q\left[q^{\mu}-\frac{q^{2}}{m_{B}^{2}-m_{K_{J}^{*}}^{2}} P^{\mu}\right],\right.
\end{aligned}
$$

which is in general analogous to the $B \rightarrow K^{*}$ ones. Here $q=P_{B}-P_{2}$, and $P=P_{B}+P_{2}$. We have the relation $2 m_{K_{J}^{*}} A_{0}(0)=\left(m_{B}+m_{K_{J}^{*}}\right) A_{1}(0)-\left(m_{B}-m_{K_{J}^{*}}\right) A_{2}(0) \quad$ in order to smear the pole at $q^{2}=0$. The polarization vector in the above equations is constructed by the $J$-rank polarization tensor

$$
\epsilon_{J \mu}(h)=\frac{1}{m_{B}^{J-1}} \epsilon_{\mu \nu_{1} \nu_{2} \ldots \nu_{J-1}}(h) P_{B}^{\nu_{1}} P_{B}^{\nu_{2}} \ldots P_{B}^{\nu_{J-1}},
$$

with $h=0, \pm 1$ being the helicity. Using the expression for $\epsilon_{\mu \nu_{1} \nu_{2} \ldots \nu_{J-1}}(h)$ which is a product of the polarization vectors with the Clebsch-Gordan coefficients, we simplify the above equation as $\epsilon_{J \mu}(h) \sim\left(\left|\vec{p}_{K^{*}}\right| / m_{K_{t}^{*}}\right)^{J-1} \tilde{\boldsymbol{\epsilon}}_{J \mu}$, with $\tilde{\epsilon}_{J \mu}(0)=\alpha_{L}^{J} \epsilon_{\mu}(0) \quad$ and $\quad \tilde{\epsilon}_{J \mu}( \pm 1)=\beta_{T}^{J} \epsilon_{\mu}( \pm 1) \quad$ and $\left|\vec{p}_{K_{J}^{*}}\right| \sim E_{K_{I}^{*}}$ in the large-recoil region. $\alpha_{L}^{J}$ and $\beta_{T}^{J}$ are products of the Clebsch-Gordan coefficients

$$
\begin{aligned}
\alpha_{L}^{J} & =C_{1,0 ; J-1,0}^{J, 0} C_{1,0 ; J-2,0}^{J-1,0} \ldots C_{1,0 ; 1,0}^{2,0}, \\
\beta_{T}^{J} & =C_{1,1 ; J-1,0}^{J, 1} C_{1,0 ; J-2,0}^{J-1,0} \ldots C_{1,0 ; 1,0}^{2,0} .
\end{aligned}
$$

The $B \rightarrow K_{J}^{*}$ form factors are nonperturbative in nature and the application of QCD theory to them mostly resorts to the Lattice QCD simulations, which is quite limited at this stage. The crucial input we use in this work is the observation that, in the heavy quark $m_{b} \rightarrow \infty$ and the large energy $E \rightarrow \infty$ limit, interactions of the heavy and light systems can be expanded in small ratios $\Lambda_{\mathrm{QCD}} / E$ and $\Lambda_{\mathrm{QCD}} / m_{B}$. At the leading power, the large energy symmetry is obtained and such symmetry to a large extent simplifies the heavy-to-light transition $[18,19]$. As a concrete application, the current $\bar{s} \Gamma b$ in QCD can be matched onto the current $\bar{s}_{n} \Gamma b_{v}$ constructed in terms of the fields in the effective theory. Here $v$ denotes the velocity of the heavy meson and $n$ is a light-like vector along the $K_{J}^{*}$ moving direction. This procedure constrains the independent Lorentz structures and reduces the seven independent hadronic form factors for each $B \rightarrow K_{J}^{*}(J \geq 1)$ type to two universal functions $\xi_{\perp}$ and $\xi_{\|}$. Explicitly, we have

$$
\begin{aligned}
& A_{0}^{K_{J}^{*}}\left(q^{2}\right)\left(\frac{\mid \vec{p}_{K_{J}^{*}}}{m_{K_{J}^{*}}}\right)^{J-1} \equiv A_{0}^{K_{J}^{*}, \mathrm{eff}} \simeq\left(1-\frac{m_{K_{J}^{*}}^{2}}{m_{B} E}\right) \xi_{\|}^{K_{J}^{*}}\left(q^{2}\right)+\frac{m_{K_{J}^{*}}}{m_{B}} \xi_{\perp}^{K_{J}^{*}}\left(q^{2}\right), \\
& A_{1}^{K_{J}^{*}}\left(q^{2}\right)\left(\frac{\left|\vec{p}_{K_{J}^{*}}\right|}{m_{K_{J}^{*}}}\right)^{J-1} \equiv A_{1}^{K_{J}^{*}, \mathrm{eff}} \simeq \frac{2 E}{m_{B}+m_{K_{J}^{*}}} \xi_{\perp}^{K_{J}^{*}}\left(q^{2}\right), \\
& A_{2}^{K_{J}^{*}}\left(q^{2}\right)\left(\frac{\left|\vec{p}_{K_{J}^{*}}\right|}{m_{K_{J}^{*}}}\right)^{J-1} \equiv A_{2}^{K_{J}^{*}, \mathrm{eff}} \simeq\left(1+\frac{m_{K_{J}^{*}}}{m_{B}}\right)\left[\xi_{\perp}^{K_{J}^{*}}\left(q^{2}\right)-\frac{m_{K_{J}^{*}}}{E} \xi_{\|}^{K_{J}^{*}}\left(q^{2}\right)\right] \text {, } \\
& V^{K_{J}^{*}}\left(q^{2}\right)\left(\frac{\left|\vec{p}_{K_{J}^{*}}\right|}{m_{K_{J}^{*}}}\right)^{J-1} \equiv V^{K_{J}^{*}, \text { eff }} \simeq\left(1+\frac{m_{K_{J}^{*}}}{m_{B}}\right) \xi_{\perp}^{K_{J}^{*}}\left(q^{2}\right), \\
& T_{1}^{K_{J}^{*}}\left(q^{2}\right)\left(\frac{\left|\vec{p}_{K_{J}^{*}}\right|}{m_{K_{J}^{*}}}\right)^{J-1} \equiv T_{1}^{K_{J}^{*}, \text { eff }} \simeq \xi_{\perp}^{K_{J}^{*}}\left(q^{2}\right), \\
& T_{2}^{K_{J}^{*}}\left(q^{2}\right)\left(\frac{\left|\vec{p}_{K_{J}^{*}}\right|}{m_{K_{J}^{*}}}\right)^{J-1} \equiv T_{2}^{K_{J}^{*}, \mathrm{eff}} \simeq\left(1-\frac{q^{2}}{m_{B}^{2}-m_{K_{J}^{*}}^{2}}\right) \xi_{\perp}^{K_{J}^{*}}\left(q^{2}\right), \\
& T_{3}^{K_{J}^{*}}\left(q^{2}\right)\left(\frac{\left|\vec{p}_{K_{J}^{*}}\right|}{m_{K_{J}^{*}}}\right)^{J-1} \equiv T_{3}^{K_{J}^{*}, \text { eff }} \simeq \xi_{\perp}^{K_{J}^{*}}\left(q^{2}\right)-\left(1-\frac{m_{K_{J}^{*}}^{2}}{m_{B}^{2}}\right) \frac{m_{K_{J}^{*}}}{E} \xi_{\|}^{K_{J}^{*}}\left(q^{2}\right) .
\end{aligned}
$$


TABLE II. Properties of the resonances $K_{J}^{*}$. The isospin symmetry relation $\mathcal{B}\left(K_{J}^{*} \rightarrow K^{-} \pi^{+}\right)=2 / 3 \mathcal{B}\left(K_{J}^{*} \rightarrow K \pi\right)$ will be used.

\begin{tabular}{llcccccc}
\hline \hline$K_{J}^{*}$ & $J^{P}$ & $n^{2 S+1} L_{J}$ & $m(\mathrm{MeV})$ & $\Gamma(\mathrm{MeV})$ & $\mathcal{B}\left(K_{J}^{*} \rightarrow K \pi\right)(\%)$ & $\alpha_{L}$ & $\beta_{T}$ \\
\hline$K^{*}(1410)$ & $1^{-}$ & $2^{3} S_{1} ?$ & $1414 \pm 15$ & $232 \pm 21$ & $6.6 \pm 1.3$ & 1 & 1 \\
$K_{0}^{*}(1430)$ & $0^{+}$ & $1^{3} P_{0}, 2^{3} P_{0} ?$ & $1425 \pm 50$ & $270 \pm 80$ & $93 \pm 10$ & 1 & - \\
$K_{2}^{*}(1430)$ & $2^{+}$ & ${ }^{3} P_{2}$ & $1432.4 \pm 1.3$ & $109 \pm 5$ & $49.9 \pm 1.2$ & $\sqrt{\frac{2}{3}}$ & $\sqrt{\frac{1}{2}}$ \\
$K^{*}(1680)$ & $1^{-}$ & $1^{3} D_{1}$ & $1717 \pm 27$ & $322 \pm 110$ & $38.7 \pm 2.5$ & 1 & 1 \\
$K_{3}^{*}(1780)$ & $3^{-}$ & $1^{3} D_{3}$ & $1776 \pm 7$ & $159 \pm 21$ & $18.8 \pm 1.0$ & $\sqrt{\frac{2}{5}}$ & $\sqrt{\frac{4}{15}}$ \\
$K_{4}^{*}(2045)$ & $4^{+}$ & $1^{3} F_{4}$ & $2045 \pm 9$ & $198 \pm 30$ & $9.9 \pm 1.2$ & $\sqrt{\frac{8}{35}}$ & $\sqrt{\frac{1}{7}}$ \\
\hline \hline
\end{tabular}

For the sake of simplicity, we will use the latter set of form factors, but as in the case of $C_{9}$ and $C_{7}$, we drop the superscript "eff" as well. In the case of $B$ to scalar meson transition, the large energy limit gives

$$
\frac{m_{B}}{m_{B}+m_{K_{0}^{*}}} F_{T}\left(q^{2}\right)=F_{1}\left(q^{2}\right)=\frac{m_{B}}{2 E} F_{0}\left(q^{2}\right)=\xi^{K_{0}^{*}}\left(q^{2}\right)
$$

The results for $\xi_{\|}^{K_{J}^{*}}$ and $\xi_{\perp}^{K_{J}^{*}}$ derived from the Bauer-StechWirbel (BSW) model [20] in Ref. [10] will be used in this work and we collect these results in Table III. For the $B \rightarrow K_{0}^{*}$ transition, it is plausible to employ $\xi^{B \rightarrow K_{0}^{*}}=$ $\xi_{\|}^{B \rightarrow K_{2}^{*}}$ since both $K_{0}^{*}$ and $K_{2}^{*}$ are p-wave states.

Several remarks on the form factors are given in order.

(i) Because of the lack of Lattice QCD simulations, the calculation of $B \rightarrow K_{J}^{*}$ form factors rely on different phenomenological models. In Table IV, as an example we show the results for the $B \rightarrow K_{2}^{*}$ form factors at $q^{2}=0$ in the ISGW2 model [21] (using the updated inputs [22]), the covariant light-front quark model $[22,23]$ and the light-cone QCD sum rules [17] and perturbative QCD approach [7] (using the light meson's light-cone distribution amplitudes [24]). From this table we can see the LEET+BSW results used here are close to the ones in the lightcone sum rules (except for $T_{3}$ ) and the perturbative QCD approach.

(ii) The large energy effective theory $[18,19]$ has neglected the interaction between the soft sector and collinear sector and it is refined by the soft-collinear effective theory [25-29], in which at leading power in $1 / m_{b}$ a form factor takes the generic expression

TABLE III. $\quad B \rightarrow K_{J}^{*}$ form factors taken from Ref. [10].

\begin{tabular}{lcc}
\hline \hline$K_{J}^{*}$ & $\xi_{\|}$ & $\xi_{\perp}$ \\
\hline$K^{*}(1410)$ & $0.22 \pm 0.03$ & $0.28 \pm 0.04$ \\
$K_{0}^{*}(1430)$ & $0.22 \pm 0.03$ & - \\
$K_{2}^{*}(1430)$ & $0.22 \pm 0.03$ & $0.28 \pm 0.04$ \\
$K^{*}(1680)$ & $0.18 \pm 0.03$ & $0.24 \pm 0.05$ \\
$K_{3}^{*}(1780)$ & $0.16 \pm 0.03$ & $0.23 \pm 0.05$ \\
$K_{4}^{*}(2045)$ & $0.13 \pm 0.03$ & $0.19 \pm 0.05$ \\
\hline \hline
\end{tabular}

$$
F_{i}\left(q^{2}\right)=C_{i} \xi\left(q^{2}\right)+C_{i}^{\prime} \int d \tau \Xi_{a}\left(\tau, q^{2}\right)
$$

Here $C_{i}$ and $C_{i}^{\prime}$ are the short-distance Wilson coefficients obtained by integrating out degrees of freedom with virtuality of $\mathcal{O}\left(m_{b}^{2}\right)$. $\xi$ is one of the above universal functions entering the large-recoil symmetries. $\Xi_{a}\left(\tau, q^{2}\right)$ is a symmetry breaking function, which can be factorized into a convolution of lightcone distribution amplitudes with the jet function. The detailed expressions of the $B \rightarrow K_{J}^{*}$ form factors (with the superscript "eff") have a similar form with the $B \rightarrow V$ transition, for instance, as Eqs. (21, 22) in Ref. [30] and, in particular, two relations for form factors remain

$$
\begin{aligned}
\frac{m_{B}}{m_{B}+m_{K_{J}^{*}}} V^{K_{J}^{*}} & =\frac{m_{B}+m_{K_{J}^{*}}}{2 E} A_{1}^{K_{J}^{*}}, \\
T_{1}^{K_{J}^{*}} & =\frac{m_{B}}{2 E} T_{2}^{K_{J}^{*}} .
\end{aligned}
$$

Again, the function $\xi$ can only be calculated in some nonperturbative QCD methods. The calculation in light-cone sum rules in conjunction with the softcollinear effective theory indicates that the $\xi$ dominates in $B \rightarrow \pi$ transition while the $\Xi_{a}$ gives corrections at the order of 5\%-10\% [31]. ${ }^{1}$ One may expect a similar size for $\Xi_{a}$ in $B \rightarrow K_{J}^{*}$ transition which will be one of the main sources of uncertainties.

(iii) It is noteworthy to point out that there are ambiguities in the internal structures of $K_{0}^{*}$, thus large discrepancies on form factors can be found in the literature. For instance, using two different assignments of $K_{0}^{*}$, namely, p-wave states without or with one unit of radial excitation, we have calculated the B to scalar meson form factors in perturbative QCD approach [34] and the results can differ up to a factor of 3 [35]. We propose that the $\mathrm{SU}(3)$ -

\footnotetext{
${ }^{1}$ A direct fit of the hadronic $B \rightarrow \pi \pi$ decay data results in a large $\Xi_{a}^{B \rightarrow \pi}$ [32], while a numerically small $\Xi_{a}^{B \rightarrow \rho}$ seems to be favored by the $B \rightarrow \rho \rho$ data [33].
} 
TABLE IV. $\quad B \rightarrow K_{2}^{*}$ form factors at $q^{2}=0$ in the ISGW2 model [21] (using the updated inputs [22]), the covariant light-front quark model [22,23] and the light-cone QCD sum rules [17] and perturbative QCD approach [7].

\begin{tabular}{|c|c|c|c|c|c|}
\hline & ISGW2 [22] & CLFQM $[22,23]$ & LCSR [17] & LEET+BSW [10] & PQCD [7] \\
\hline$V^{B K_{2}^{*}}$ & 0.38 & 0.29 & $0.16 \pm 0.02$ & $0.21 \pm 0.03$ & $0.21_{-0.05}^{+0.06}$ \\
\hline$A_{0}^{B K_{2}^{*}}$ & 0.27 & 0.23 & $0.25 \pm 0.04$ & $0.15 \pm 0.02$ & $0.18_{-0.04}^{+0.05}$ \\
\hline$A_{1}^{B K_{2}^{*}}$ & 0.24 & 0.22 & $0.14 \pm 0.02$ & $0.14 \pm 0.02$ & $0.13_{-0.03}^{+0.04}$ \\
\hline$A_{2}^{B K_{2}^{*}}$ & 0.22 & 0.21 & $0.05 \pm 0.02$ & $0.14 \pm 0.02$ & $0.08_{-0.02}^{+0.03}$ \\
\hline$T_{1}^{B K_{2}^{*}}$ & & 0.28 & $0.14 \pm 0.02$ & $0.16 \pm 0.02$ & $0.17_{-0.04}^{+0.05}$ \\
\hline$T_{3}^{B K_{2}^{*}}$ & & -0.25 & $0.01_{-0.01}^{+0.02}$ & $0.10 \pm 0.02$ & $0.14_{-0.03}^{+0.05}$ \\
\hline
\end{tabular}

symmetry related processes can be used to pin down the uncertainties. Channels of this type include the semileptonic $B \rightarrow a_{0}(1450) l \bar{\nu}$, $B_{s} \rightarrow K_{0}^{*} l \bar{\nu}$ decays and the exclusive channels $\bar{B}^{0} \rightarrow a_{0}^{+}(1450) D_{s}^{-} / D^{-}, \quad B^{-} \rightarrow a_{0}^{0}(1450) D_{s}^{-}$, $\bar{B}_{s} \rightarrow K_{0}^{*+} D^{-}$. Semileptonic decays provide the information of form factors in the full kinematics region through the differential decay width distribution. The above exclusive processes are color-favored and free of annihilation diagrams; therefore the factorization method works phenomenologically well for them. In the factorization context, the decay amplitudes, taking $\bar{B}^{0} \rightarrow$ $D_{s}^{-} a_{0}^{+}(1450)$ as an example, are written as

$$
\begin{aligned}
\mathcal{A}\left(\bar{B}^{0} \rightarrow D_{s}^{-} a_{0}^{+}\right)= & \frac{i G_{F}}{\sqrt{2}} i V_{c s}^{*} V_{u b} a_{1} f_{D_{s}} \\
& \times\left(m_{B}^{2}-m_{D_{s}}^{2}\right) F_{0}^{B \rightarrow a_{0}}\left(m_{D_{s}}^{2}\right),
\end{aligned}
$$

where $a_{1} \sim 1$ being the Wilson coefficients and $f_{D_{s}}$ denoting the decay constant of the $D_{s}$ meson. In particular, most of the inputs will be canceled if the ratio

$$
r=\frac{\Gamma\left(\bar{B}^{0} \rightarrow D_{s}^{-} a_{0}^{+}\right)}{\Gamma\left(\bar{B}^{0} \rightarrow D_{s}^{-} \pi^{+}\right)} \simeq \frac{\left[F_{0}^{B \rightarrow a_{0}}\left(m_{D_{s}}^{2}\right)\right]^{2}}{\left[F_{0}^{B \rightarrow \pi}\left(m_{D_{s}}^{2}\right)\right]^{2}}
$$

is considered. The decay $\bar{B}^{0} \rightarrow D_{s}^{-} \pi^{+}$has a quite large branching ratio $\mathcal{B}=(2.4 \pm 0.4) \times 10^{-5}$ [14]. The measurement of $\bar{B}^{0} \rightarrow D_{s}^{-} a_{0}^{+}$in the future will consequently determine the $F_{0}^{B \rightarrow a_{0}}$ and also $F_{0}^{B \rightarrow K_{0}^{*}}$ up to $\mathrm{SU}(3)$ symmetry breaking effects. Replacing $D_{s}^{-}$by $D_{s}^{*-}$, one can extract the form factor $F_{1}^{B \rightarrow a_{0} / K_{0}^{*}}$ from the relevant data in future.

\section{DIFFERENTIAL DECAY DISTRIBUTIONS AND FORWARD-BACKWARD ASYMMETRIES}

The convention on the kinematics in $B \rightarrow K_{J}^{*}(\rightarrow$ $K \pi) l^{+} l^{-}$is illustrated in Fig. 1. The moving direction of
$K_{J}^{*}$ in the $B$ rest frame is chosen as the $z$ axis. The polar angle $\theta_{K}\left(\theta_{l}\right)$ is defined as the angle between the flight direction of $K^{-}\left(\mu^{-}\right)$and the $z$ axis in the $K_{J}^{*}$ (lepton pair) rest frame. $\phi$ is the angle defined by the decay planes of $K_{J}^{*}$ and the lepton pair.

$B \rightarrow K_{J}^{*}(\rightarrow K \pi) l^{+} l^{-}$is a quasi four-body decay process and proceeds via three steps: $B$ meson first decays into a nearly onshell strange meson plus a pair of leptons; the $K_{J}^{*}$ meson propagates followed by its strong decay into the $K \pi$ state. The decay amplitudes of $B \rightarrow\left(K^{-} \pi^{+}\right) l^{+} l^{-}$are obtained by sandwiching Eq. (1) between the initial and final states, in which the spinor product $[\bar{s} b]$ by hadronic matrix element will be replaced by hadronic form factors. The operator realization of this picture is

$$
\begin{aligned}
& \left\langle l^{+} l^{-}|[\bar{l} l]| 0\right\rangle\left\langle K \pi|[\bar{s} b]| \bar{B}^{0}\right\rangle \\
& \quad \simeq\left\langle l^{+} l^{-}|[\bar{l} l]| 0\right\rangle \int d^{4} p_{K_{J}^{*}} \frac{\left\langle K \pi \mid K_{J}^{*}\right\rangle\left\langle K_{J}^{*}|[\bar{s} b]| \bar{B}^{0}\right\rangle}{p_{K_{J}^{*}}^{2}-m_{K_{J}^{*}}^{2}+i m_{K_{J}^{*}} \Gamma_{K_{J}^{*}}},
\end{aligned}
$$

with $p_{K_{J}^{*}}^{2}=m_{K \pi}^{2}$. In appendix $\mathrm{A}$, we will compute the required quantities in the three steps with the use of helicity amplitudes. Combining the individual pieces, we obtain the angular distributions

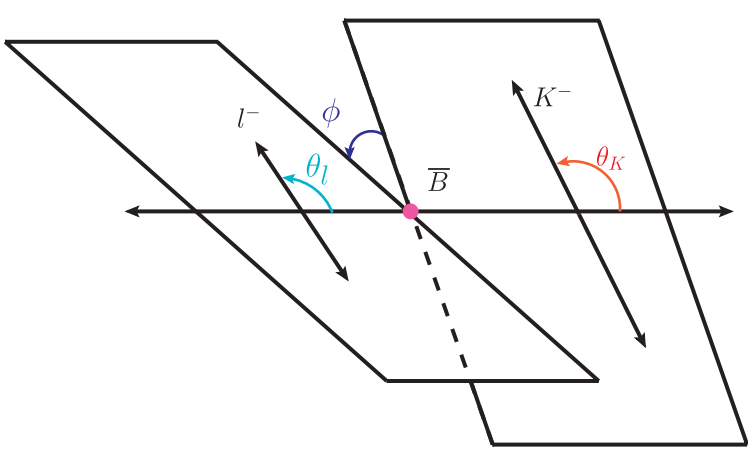

FIG. 1 (color online). Kinematics in $\bar{B} \rightarrow \bar{K}_{J}^{*}\left(\rightarrow K^{-} \pi^{+}\right) l^{+} l^{-}$. $K_{J}^{*}$ moves along the $z$ axis in the $B$ rest frame. $\theta_{K}\left(\theta_{l}\right)$ is defined in $K_{J}^{*}$ (lepton pair) rest frame as the angle between $z$-axis and the flight direction of $K^{-}\left(\mu^{-}\right)$, respectively. The azimuth angle $\phi$ is the angle between the $K_{J}^{*}$ decay and lepton pair planes. 


$$
\begin{aligned}
& \frac{d^{4} \Gamma}{d m_{K \pi}^{2} d q^{2} d \cos \theta_{K} d \cos \theta_{l} d \phi} \\
& =\left[I_{1}^{c}+2 I_{1}^{s}+\left(I_{2}^{c}+2 I_{2}^{s}\right) \cos \left(2 \theta_{l}\right)+2 I_{3} \sin ^{2} \theta_{l} \cos (2 \phi)\right. \\
& \quad+2 \sqrt{2} I_{4} \sin \left(2 \theta_{l}\right) \cos \phi+2 \sqrt{2} I_{5} \sin \left(\theta_{l}\right) \cos \phi \\
& \quad+2 I_{6} \cos \theta_{l}+2 \sqrt{2} I_{7} \sin \left(\theta_{l}\right) \sin \phi \\
& \left.+2 \sqrt{2} I_{8} \sin \left(2 \theta_{l}\right) \sin \phi+2 I_{9} \sin ^{2} \theta_{l} \sin (2 \phi)\right]
\end{aligned}
$$

with the angular coefficients

$$
\begin{aligned}
I_{1}^{c}= & \left(\left|A_{L 0}\right|^{2}+\left|A_{R 0}\right|^{2}\right)+8 \frac{m_{l}^{2}}{q^{2}} \operatorname{Re}\left[A_{L 0} A_{R 0}^{*}\right]+4 \frac{m_{l}^{2}}{q^{2}}\left|A_{t}\right|^{2}, \\
I_{1}^{s}= & \frac{3}{4}\left[\left|A_{L \perp}\right|^{2}+\left|A_{L \|}\right|^{2}+\left|A_{R \perp}\right|^{2}+\left|A_{R \|}\right|^{2}\right]\left(1-\frac{4 m_{l}^{2}}{3 q^{2}}\right) \\
& +\frac{4 m_{l}^{2}}{q^{2}} \operatorname{Re}\left[A_{L \perp} A_{R \perp}^{*}+A_{L \|} A_{R \|}^{*}\right], \\
I_{2}^{c}= & -\beta_{l}^{2}\left(\left|A_{L 0}\right|^{2}+\left|A_{R 0}\right|^{2}\right), \\
I_{2}^{s}= & \frac{1}{4} \beta_{l}^{2}\left(\left|A_{L \perp}\right|^{2}+\left|A_{L \|}\right|^{2}+\left|A_{R \perp}\right|^{2}+\left|A_{R \|}\right|^{2}\right), \\
I_{3}= & \frac{1}{2} \beta_{l}^{2}\left(\left|A_{L \perp}\right|^{2}-\left|A_{L \|}\right|^{2}+\left|A_{R \perp}\right|^{2}-\left|A_{R\|\|}\right|^{2}\right), \\
I_{4}= & \frac{1}{\sqrt{2}} \beta_{l}^{2}\left[\operatorname{Re}\left(A_{L 0} A_{L \|}^{*}\right)+\operatorname{Re}\left(A_{R 0} A_{R \|}^{*}\right],\right. \\
I_{5}= & \sqrt{2} \beta_{l}\left[\operatorname{Re}\left(A_{L 0} A_{L \perp}^{*}\right)-\operatorname{Re}\left(A_{R 0} A_{R \perp}^{*}\right)\right], \\
I_{6}= & 2 \beta_{l}\left[\operatorname{Re}\left(A_{L \|} A_{L \perp}^{*}\right)-\operatorname{Re}\left(A_{R \|} A_{R \perp}^{*}\right)\right], \\
I_{7}= & \sqrt{2} \beta_{l}\left[\operatorname{Im}\left(A_{L 0} A_{L \|}^{*}\right)-\operatorname{Im}\left(A_{R 0} A_{R \|}^{*}\right)\right], \\
I_{8}= & \frac{1}{\sqrt{2}} \beta_{l}^{2}\left[\operatorname{Im}\left(A_{L 0} A_{L \perp}^{*}\right)+\operatorname{Im}\left(A_{R 0} A_{R \perp}^{*}\right)\right], \\
I_{9}= & \beta_{l}^{2}\left[\operatorname{Im}\left(A_{L \|} A_{L \perp}^{*}\right)+\operatorname{Im}\left(A_{R \|} A_{R \perp}^{*}\right)\right] .
\end{aligned}
$$

The lepton mass correction factor is $\beta_{l}=\sqrt{1-4 m_{l}^{2} / q^{2}}$. The functions $A_{L / R i}$ are defined by

$$
\begin{aligned}
& A_{L / R 0 / t}=\sum_{J=0,1,2 \ldots} \sqrt{N_{K_{J}^{*}}} Y_{J}^{0}(\theta, 0) \mathcal{M}_{B}\left(K_{J}^{*}, L / R, 0 / t\right)
\end{aligned}
$$

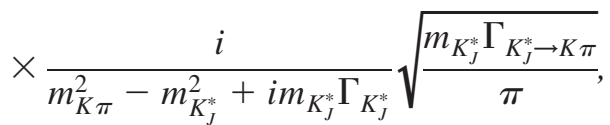

$$
\begin{aligned}
& A_{L / R \| / \perp}=\sum_{J=0,1,2 \ldots} \sqrt{N_{K_{J}^{*}}} Y_{J}^{-1}(\theta, 0) \mathcal{M}_{B}\left(K_{J}^{*}, L / R, \| / \perp\right) \\
& \times \frac{i}{m_{K \pi}^{2}-m_{K_{J}^{*}}^{2}+i m_{K_{J}^{*}} \Gamma_{K_{J}^{*}}} \sqrt{\frac{m_{K_{J}^{*} \Gamma_{K_{J}^{*} \rightarrow K \pi}}}{\pi},}
\end{aligned}
$$

with $N_{K_{J}^{*}}=\frac{\sqrt{\lambda} q^{2} \beta_{l}}{256 \pi^{3} m_{B}^{3}} \cdot \mathcal{M}_{B}$ is the decay amplitudes of $B \rightarrow$ $K_{J}^{*} V$ to be given in the appendix. In the narrow-width limit, the integration over the $K \pi$ invariant mass will be conducted as

$$
\int d m_{K \pi}^{2} \frac{m_{K_{J}^{*}} \Gamma_{K_{J}^{*}}}{\pi} \frac{1}{\left(m_{K \pi}^{2}-m_{K_{J}^{*}}^{2}\right)^{2}+m_{K_{J}^{*}}^{2} \Gamma_{K_{J}^{*}}^{2}}=1 .
$$

Integrating out the angles $\theta_{l}, \theta_{K}$ and $\phi$, we obtain the dilepton mass spectrum

$\frac{d^{2} \Gamma_{L}}{d q^{2} d m_{K \pi}^{2}}=\frac{2}{3} \int_{-1}^{1} 2 \pi d \cos \theta_{K}\left(3 I_{1}^{c}-I_{2}^{c}\right)$,

$\frac{d^{2} \Gamma_{T}}{d q^{2} d m_{K \pi}^{2}}=\frac{2}{3} \int_{-1}^{1} 2 \pi d \cos \theta_{K}\left(6 I_{1}^{s}-2 I_{2}^{s}\right)$,

$\frac{d^{2} \Gamma}{d q^{2} d m_{K \pi}^{2}}=\frac{d^{2} \Gamma_{L}}{d q^{2} d m_{K \pi}^{2}}+\frac{d^{2} \Gamma_{T}}{d q^{2} d m_{K \pi}^{2}}$,

and its expression in the massless limit

$$
\frac{d^{2} \Gamma_{i}}{d q^{2} d m_{K \pi}^{2}}=\frac{8}{3} \int_{-1}^{1} 2 \pi d \cos \theta_{K}\left(\left|A_{L i}\right|^{2}+\left|A_{R i}\right|^{2}\right)
$$

with $i=0, \pm 1$ or $i=0, \perp, \|$.

The differential FBA in this process is defined by

$$
\begin{aligned}
\frac{d^{2} A_{\mathrm{FB}}}{d q^{2} d m_{K \pi}^{2}} & =\left[\int_{0}^{1}-\int_{-1}^{0}\right] d \cos \theta_{l} \frac{d^{3} \Gamma}{d q^{2} d \cos \theta_{l} d m_{K \pi}^{2}} \\
& =\frac{2}{3} \int_{-1}^{1} 2 \pi \cos \theta_{K} 3 I_{6},
\end{aligned}
$$

while the normalized differential FBA is given by

$$
\begin{aligned}
\frac{\overline{d^{2} A_{\mathrm{FB}}}}{d q^{2} d m_{K \pi}^{2}} & =\frac{\frac{d^{2} A_{\mathrm{FB}}}{d q^{2} d m_{K \pi}^{2}}}{\frac{d^{2} \Gamma}{d q^{2} d m_{K \pi}^{2}}} \\
& =\frac{\int_{-1}^{1} d \cos \theta_{K} 3 I_{6}}{\int_{-1}^{1} d \cos \theta_{K}\left[3 I_{1}^{c}+6 I_{1}^{s}-I_{2}^{c}-2 I_{2}^{s}\right]}
\end{aligned}
$$

\section{NUMERICAL RESULTS AND DISCUSSIONS}

Before presenting the numerical results, we start with an estimate of the contributions from different mesons. It is noticed that larger the $J$ is the smaller is the contribution. (1) The $K_{J}^{*}$ with a larger spin is heavier and thus the phase space is smaller. (2) The Clebsch-Gordan coefficient products $\alpha_{L}^{J}$ and $\beta_{T}^{J}$ decrease with the increase of $J$. (3) The $B \rightarrow K_{J}^{*}$ form factors suppress the heavier $K_{J}^{*}$ further. Moreover, the tiny branching ratios of $K^{*}(1410)$ and $K_{4}^{*}(2045)$ into $K^{-} \pi^{+}$result in very smaller effects. As a consequence we find that the $K_{4}^{*}(2045)$ is negligibly small.

We plot the differential branching ratios $\frac{d^{2} B R_{i}}{d q^{2} d m_{K \pi}^{2}}$ (in units of $10^{-8} \mathrm{GeV}^{-4}$ ), with the subscript $i$ denoting the total, longitudinal and transverse polarizations) and the 

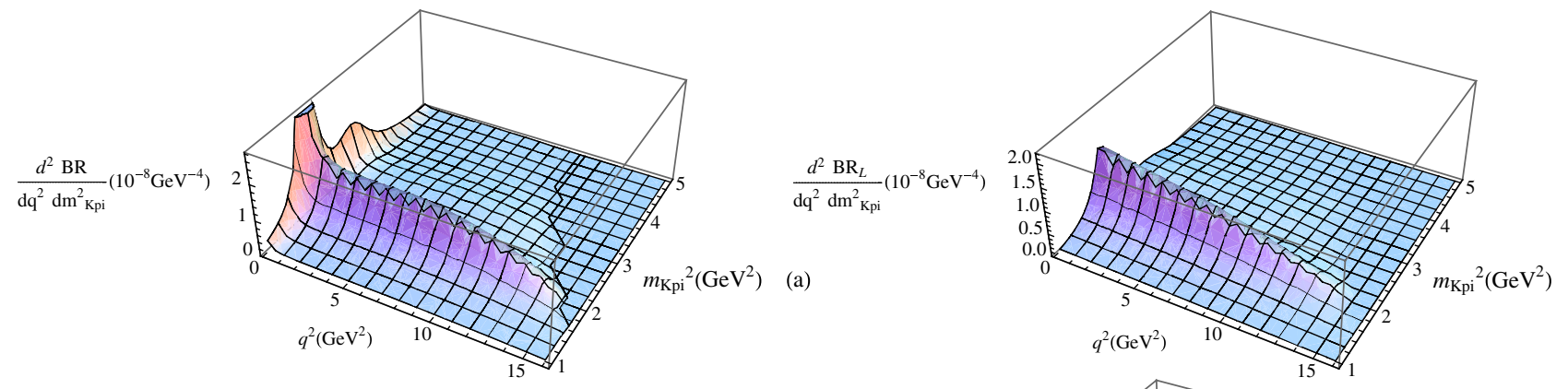

(b)
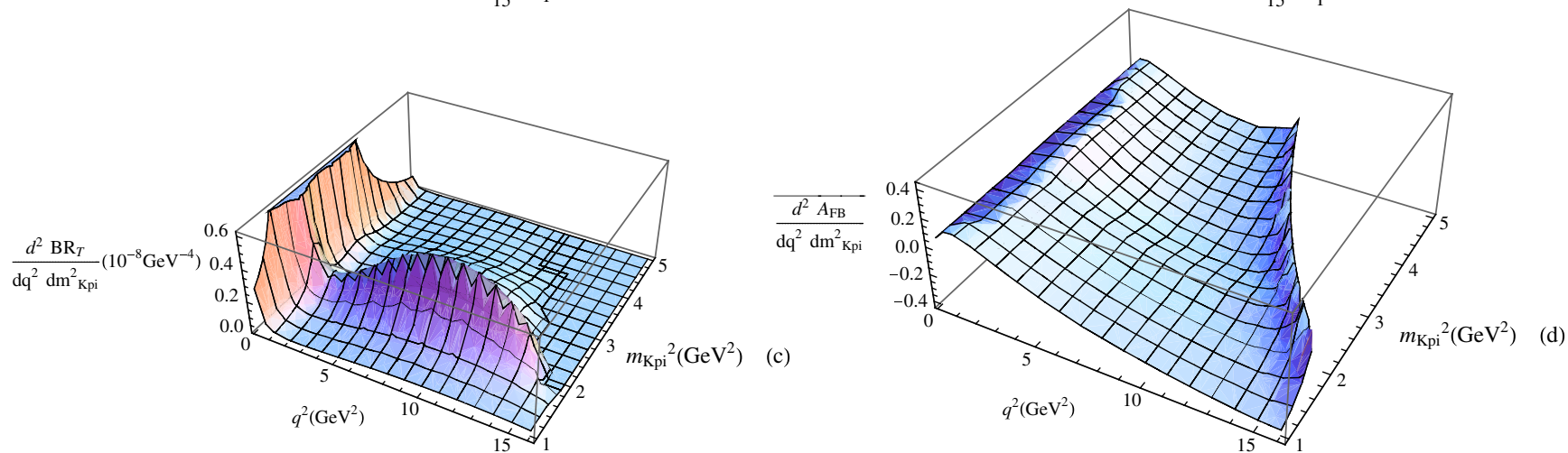

FIG. 2 (color online). Differential branching ratios $\frac{d^{2} B R_{i}}{d q^{2} d m_{K \pi}^{2}}$, with $i$ denoting the total (a), longitudinal (b) and transverse polarizations (c), and the normalized FBA $\frac{\overline{d^{2} A_{\mathrm{FB}}}}{d q^{2} d m_{K \pi}^{2}}$ (d) for $\bar{B}^{0} \rightarrow K^{-} \pi^{+} \mu^{+} \mu^{-}$in the mass region $1 \mathrm{GeV}^{2}<m_{K \pi}^{2}<5 \mathrm{GeV}^{2}$.
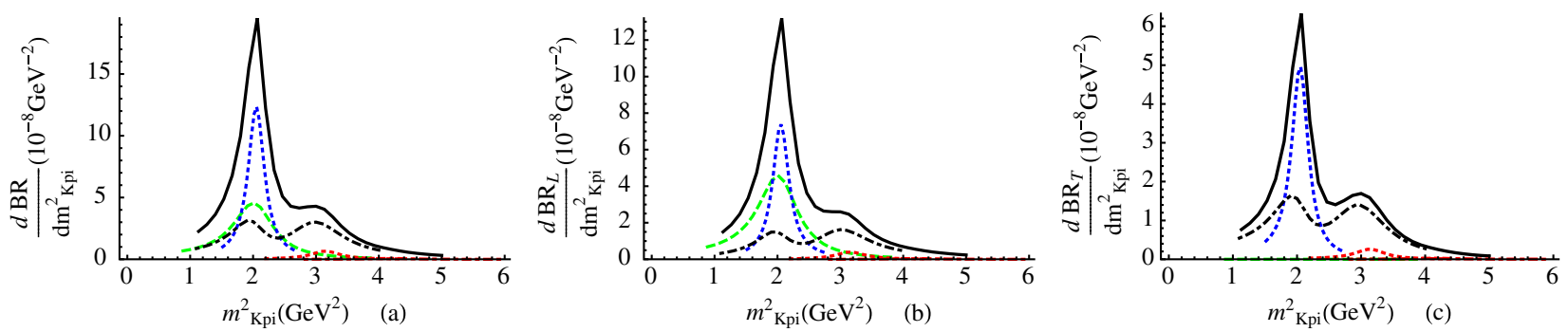

FIG. 3 (color online). Differential branching ratios $\frac{d B R_{i}}{d m_{K}^{2}}\left(\bar{B}^{0} \rightarrow K^{-} \pi^{+} \mu^{+} \mu^{-}\right)$integrated over the kinematics region $q^{2}>4 m_{l}^{2}$. The black (solid) curve denotes the total contribution, while individual terms are given by the green (dashed) line for $K_{0}^{*}(1430)$, blue dotted line for $K_{2}^{*}(1430)$, the black (dot-dashed) line for $K^{*}(1680)$ and $K^{*}(1410)$ with the interference incorporated, and the red (dotted) curve with a very small magnitude for $K_{3}^{*}(1780)$. The contribution from $K_{4}^{*}(2045)$ is negligibly small.

normalized FBA $\frac{\overline{d^{2} A_{\mathrm{FB}}}}{d q^{2} d m_{K \pi}^{2}}$ for $\bar{B}^{0} \rightarrow K^{-} \pi^{+} \mu^{+} \mu^{-}$in Fig. 2. By integrating the differential distributions over $q^{2}$, we obtain their dependence on $m_{K \pi}^{2}$. Figure 3 shows the differential branching ratios $\frac{d B R_{i}}{d m_{K \pi}^{2}}\left(\bar{B}^{0} \rightarrow K^{-} \pi^{+} \mu^{+} \mu^{-}\right)$ (in units of $10^{-8} \mathrm{GeV}^{-2}$ ) integrated over the kinematics region $4 m_{l}^{2}<q^{2}<\left(m_{B}-m_{K \pi}\right)^{2}$, while Fig. 4 gives the results under the integration over $1 \mathrm{GeV}^{2}<q^{2}<6 \mathrm{GeV}^{2}$. In these figures, the black (solid) curve denotes the total contribution, while individual terms are given by the green (dashed) line for $K_{0}^{*}(1430)$, blue dotted line for $K_{2}^{*}(1430)$, the black (dot-dashed) line for $K^{*}(1680)$ and $K^{*}(1410)$ with the interference incorporated, and the red (dotted) curve with a very small magnitude for $K_{3}^{*}(1780)$. The contribution from $K_{4}^{*}(2045)$ is negligibly small. From these figures, we can see that for $m_{K \pi}^{2}=2 \mathrm{GeV}^{2}$, the $K_{2}^{*}$ dominates; while at $m_{K \pi}^{2} \simeq 3 \mathrm{GeV}^{2}$, the $B \rightarrow K^{*}(1680)$ contribution is the largest, especially in the transverse polarization.

Now let us analyze the zero crossing point $s_{0}$ of FBAs satisfying $\left.\frac{d^{2} A_{\mathrm{FB}}}{d q^{2} d m_{K \pi}^{2}}\right|_{q^{2}=s_{0}}=0$ and governed by the equation

$$
\begin{aligned}
& \operatorname{Re}\left[C_{9}\right] A_{1}\left(s_{0}\right) V\left(s_{0}\right)+C_{7 L} \frac{m_{b}\left(m_{B}+m_{K_{J}^{*}}\right)}{s_{0}} A_{1}\left(s_{0}\right) T_{1}\left(s_{0}\right) \\
& \quad+C_{7 L} \frac{m_{b}\left(m_{B}-m_{K_{J}^{*}}\right)}{s_{0}} T_{2}\left(s_{0}\right) V\left(s_{0}\right)=0 .
\end{aligned}
$$



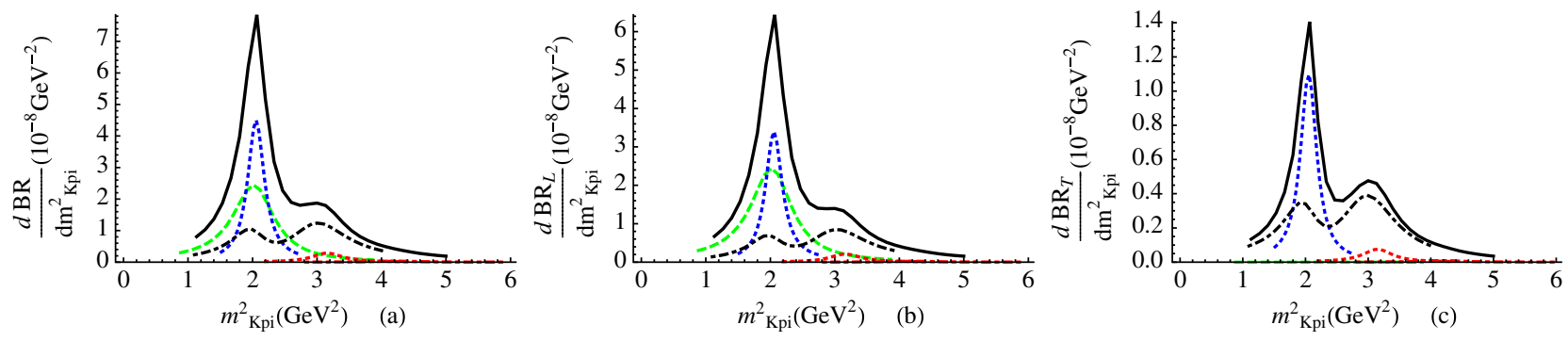

FIG. 4 (color online). Same as Fig. 3, but integrated over $1 \mathrm{GeV}^{2}<q^{2}<6 \mathrm{GeV}^{2}$.

Substituting the relations from the large energy limit into the above equation, we find that the dependence on the form factors cancels completely and more explicitly Eq. (20) gives

$$
s_{0}=(3.1 \pm 0.1) \mathrm{GeV}^{2},
$$

where the uncertainties are caused by $m_{K_{J}^{*}}^{2} / m_{B}^{2}$ corrections in the form factor relations in Eq. (7). As we have discussed in Sec. II, the interaction of collinear and soft sectors brings in symmetry breaking effects. After the inclusion of them, only two relations among form factors remain as in Eq. (10). Define the ratio

$$
\mathcal{R}^{K_{J}^{*}}\left(q^{2}\right) \equiv \frac{m_{B}+m_{K_{J}^{*}}}{m_{B}} \frac{T_{1}^{K_{J}^{*}}\left(q^{2}\right)}{V^{K_{J}^{*}}\left(q^{2}\right)},
$$

we find that Eq. (20) becomes

$$
\operatorname{Re}\left[C_{9}\right]+2 \frac{m_{b} m_{B}}{s_{0}} C_{7 L} \mathcal{R}^{K_{J}^{*}}\left(s_{0}\right)=0 .
$$

The analysis in Ref. [30] indicates that the ratio $\mathcal{R}$ can deviate from 1 by $10 \%$ in the $B \rightarrow V$ transition (see Eq. (124) of [30]). Using the PQCD [7] and LCSR [17] results for the $B \rightarrow K_{2}$ form factors, we have

$$
\mathcal{R}_{\mathrm{PQCD}}^{K_{2}} \sim 1.03, \quad \mathcal{R}_{\mathrm{LCSR}}^{K_{2}} \sim 1.11,
$$

where the $q^{2}$-dependence is negligible since form factors $T_{1}$ and $V$ are found to have similar $q^{2}$-distribution in both model calculations. Suppose that the $\mathcal{R}^{K_{J}^{*}}$ deviates from 1 by $10 \%$, the $s_{0}$ is also shifted by roughly $10 \%$, namely $0.3 \mathrm{GeV}^{2}$.

Our analysis of the $m_{K \pi}$ dependence can be generalized to similar channels such as $\bar{B}^{0} \rightarrow J / \psi K_{J}^{*} \rightarrow\left(\mu^{+} \mu^{-}\right) \times$ $\left(K^{-} \pi^{+}\right)$and $B_{s} \rightarrow f_{J}\left(\rightarrow K^{+} K^{-}\right) l^{+} l^{-}$. For the former processes, however, apart from the $B \rightarrow K_{J}^{*}$ form factors, it is likely that the effective Wilson coefficients $a_{2}$ depends on the spin of $K_{J}^{*}$ as well (for a recent discussion see Ref. [36]). Although the relative strengths among $K_{J}^{*}$ may be modified, the structure of the dependence on $m_{K \pi}^{2}$ is expected to be similar. For the latter, uncertainties are presumably smaller, as a recent measurement of $B_{s} \rightarrow$ $J / \psi K^{+} K^{-}$clearly shows the peak at $f_{2}^{\prime}(1525)$ [37].

\section{CONCLUSION}

In summary, we have analyzed the resonant contributions in the process $\bar{B}^{0} \rightarrow K^{-} \pi^{+} \mu^{+} \mu^{-}$with the $K^{-} \pi^{+}$ invariant mass square $m_{K \pi}^{2} \in[1,5] \mathrm{GeV}^{2}$. Width effects of the strange mesons involved in this range, $K^{*}(1410)$, $K_{0}^{*}(1430), K_{2}^{*}(1430), K^{*}(1680), K_{3}^{*}(1780)$ and $K_{4}^{*}(2045)$, are incorporated. In terms of the helicity amplitudes, we derive a compact form for the full angular distributions, through which the branching ratios, forward-backward asymmetries and polarizations are attained. To pin down the uncertainties in the form factors, we suggest the measurements of a set of SU(3)-related processes which are useful. Using the form factors from the large energy limit, we derive the dependence of the branching fractions on $m_{K \pi}$, and we point out that the $K_{2}^{*}$ and $K^{*}(1680)$ contributions can be separated from the rest, in particular, in the transverse polarizations. The generalization into $\bar{B} \rightarrow$ $J / \psi K_{J}^{*}\left(\rightarrow K^{-} \pi^{+}\right)$and $B_{s} \rightarrow f_{J}\left(\rightarrow K^{+} K^{-}\right) l^{+} l^{-}$is also discussed briefly.

\section{ACKNOWLEDGMENTS}

W.W. is grateful to Ulrik Egede for the enlightening discussions on the interferences of $K_{J}^{*}$ which initiated this work. We thank Ahmed Ali for fruitful discussions, and Run-Hui Li for the collaboration at the early stage of this work. This work is supported by National Natural Science Foundation of China under the Grant No. 10735080 and 11075168 (C. D. Lü) and the Alexander von Humboldt Stiftung (W. Wang).

\section{APPENDIX: HELICITY AMPLITUDES}

The differential distributions are divided into several individual pieces and each of them can be expressed in terms of the helicity amplitudes which are Lorentz invariant.

(i) $B$ decays into $K_{J}^{*}$

The spin- $0 K_{0}^{*}$ in the final state has only one polarization state and the longitudinal amplitudes are 


$$
\begin{aligned}
& i \mathcal{M}_{B}\left(K_{0}^{*}, L / R, 0\right)=N_{1} i\left[\left(C_{9} \mp C_{10}\right) \frac{\sqrt{\lambda}}{\sqrt{q^{2}}} F_{1}\left(q^{2}\right)+2\left(C_{7 L}-C_{7 R}\right) \frac{\sqrt{\lambda} m_{b}}{\sqrt{q^{2}}\left(m_{B}+m_{K_{0}^{*}}\right)} F_{T}\left(q^{2}\right)\right], \\
& i \mathcal{M}_{B}\left(K_{0}^{*}, L / R, t\right)=N_{1} i\left[\left(C_{9} \mp C_{10}\right) \frac{m_{B}^{2}-m_{K_{0}^{*}}^{2}}{\sqrt{q^{2}}} F_{0}\left(q^{2}\right)\right],
\end{aligned}
$$

with $N_{1}=\frac{i G_{F}}{4 \sqrt{2}} \frac{\alpha_{\mathrm{em}}}{\pi} V_{t b} V_{t s}^{*}$. The function $\lambda$ is related to the magnitude of the $K_{J}^{*}$ momentum in $B$ meson rest frame: $\lambda \equiv$ $\lambda\left(m_{B}^{2}, m_{K_{J}^{*}}^{2}, q^{2}\right) \stackrel{\pi}{=} 2 m_{B}\left|\vec{p}_{K_{I}^{*}}\right|$, and $\lambda\left(a^{2}, b^{2}, c^{2}\right)=\left(a^{2}-b^{2}-c^{2}\right)^{2}-4 b^{2} c^{2}$. Here the script $t$ denotes the timelike component of a virtual vector/axial-vector meson decays into a lepton pair. In the case of strange mesons with spin $J \geq 1$, the $K^{-} \pi^{+}$system can be either longitudinally or transversely polarized:

$$
\begin{aligned}
i \mathcal{M}_{B}\left(K_{J}^{*}, L, 0\right)= & \frac{\alpha_{L}^{J} N_{1} i}{2 m_{K_{J}^{*}} \sqrt{q^{2}}}\left[\left(C_{9}-C_{10}\right)\left[\left(m_{B}^{2}-m_{K_{J}^{*}}^{2}-q^{2}\right)\left(m_{B}+m_{K_{J}^{*}}\right) A_{1}-\frac{\lambda}{m_{B}+m_{K_{J}^{*}}} A_{2}\right]\right. \\
& \left.+2 m_{b}\left(C_{7 L}-C_{7 R}\right)\left[\left(m_{B}^{2}+3 m_{K_{J}^{*}}^{2}-q^{2}\right) T_{2}-\frac{\lambda}{m_{B}^{2}-m_{K_{J}^{*}}^{2}} T_{3}\right]\right], i \mathcal{M}_{B}\left(K_{J}^{*}, L, \pm\right) \\
= & \beta_{T}^{J} N_{1} i\left[\left(C_{9}-C_{10}\right)\left[\left(m_{B}+m_{K_{J}^{*}}\right) A_{1} \mp \frac{\sqrt{\lambda}}{m_{B}+m_{K_{J}^{*}}} V\right]-\frac{2 m_{b}\left(C_{7 L}+C_{7 R}\right)}{q^{2}}\left( \pm \sqrt{\lambda} T_{1}\right)\right. \\
& \left.+\frac{2 m_{b}\left(C_{7 L}-C_{7 R}\right)}{q^{2}}\left(m_{B}^{2}-m_{K_{J}^{*}}^{2}\right) T_{2}\right], \\
& i \mathcal{M}_{B}\left(K_{J}^{*}, L, t\right)=\alpha_{L}^{J} i N_{1}\left(C_{9}-C_{10}\right) \frac{\sqrt{\lambda}}{\sqrt{q^{2}}} A_{0} .
\end{aligned}
$$

For the sake of convenience, we define

$$
\begin{aligned}
i \mathcal{M}_{B}\left(K^{*}, L, \perp / \|\right) & =\frac{1}{\sqrt{2}}\left[i \mathcal{M}_{B}\left(K^{*}, L,+\right) \mp i \mathcal{M}_{B}\left(K^{*}, L,-\right)\right] \\
i \mathcal{M}_{B}\left(K^{*}, L, \perp\right) & =-i \beta_{T}^{J} \sqrt{2} N_{1}\left[\left(C_{9}-C_{10}\right) \frac{\sqrt{\lambda} V}{m_{B}+m_{K_{J}^{*}}}+\frac{2 m_{b}\left(C_{7 L}+C_{7 R}\right)}{q^{2}} \sqrt{\lambda} T_{1}\right] \\
i \mathcal{M}_{B}\left(K^{*}, L, \|\right) & =i \beta_{T}^{J} \sqrt{2} N_{1}\left[\left(C_{9}-C_{10}\right)\left(m_{B}+m_{K_{J}^{*}}\right) A_{1}+\frac{2 m_{b}\left(C_{7 L}-C_{7 R}\right)}{q^{2}}\left(m_{B}^{2}-m_{K_{J}^{*}}^{2}\right) T_{2}\right]
\end{aligned}
$$

The right-handed decay amplitudes are defined in a similar way

$$
A_{R i}=\left.A_{L i}\right|_{C_{10} \rightarrow-C_{10}} \text {. }
$$

The combination of the timelike decay amplitude is used in the differential distribution

$$
\begin{gathered}
i \mathcal{M}_{B}\left(K_{0}^{*}, t\right)=i \mathcal{M}_{B}\left(K_{0}^{*}, R, t\right)-i \mathcal{M}_{B}\left(K_{0}^{*}, L, t\right)=2 \alpha_{L}^{J} i C_{10} N_{1} \frac{m_{B}^{2}-m_{K_{0}^{*}}^{2}}{\sqrt{q^{2}}} F_{0}\left(q^{2}\right), \\
i \mathcal{M}_{B}\left(K_{J}^{*}, t\right)=i \mathcal{M}_{B}\left(K_{J}^{*}, R, t\right)-i \mathcal{M}_{B}\left(K_{J}^{*}, L, t\right)=2 \alpha_{L}^{J} i N_{1} C_{10} \frac{\sqrt{\lambda}}{\sqrt{q^{2}}} A_{0}\left(q^{2}\right)
\end{gathered}
$$

(ii) Nonzero leptonic amplitudes are given as follows

$$
\begin{aligned}
\mathcal{M}_{L, R}\left(\lambda_{l}, \lambda_{\bar{l}}, \lambda_{V}\right) & =T_{\lambda_{l}, \lambda_{l}}^{L, R} D_{\lambda_{V}, \lambda_{l}-\lambda_{\bar{l}}}^{1 *}\left(\phi, \pi-\theta_{l}, 0\right), \\
\mathcal{M}_{L}\left(\frac{1}{2}, \frac{1}{2}, t\right) & =-\mathcal{M}_{L}\left(\frac{-1}{2}, \frac{-1}{2}, t\right)=-\mathcal{M}_{R}\left(\frac{1}{2}, \frac{1}{2}, t\right)=\mathcal{M}_{R}\left(\frac{-1}{2}, \frac{-1}{2}, t\right)=-2 m_{l} .
\end{aligned}
$$

with $q_{ \pm}=\sqrt{q^{2}} \pm \sqrt{q^{2}-4 m_{l}^{2}}$. The reduced matrix elements are given as 


$$
\begin{aligned}
T_{(1 / 2)(1 / 2)}^{L} & =T_{(-1 / 2)(-1 / 2)}^{L}=T_{(1 / 2)(1 / 2)}^{R}=T_{(-1 / 2)(-1 / 2)}^{R}=-2 m_{l}, \\
T_{(1 / 2)(-1 / 2)}^{L} & =T_{(-1 / 2)(1 / 2)}^{R}=\sqrt{2} q_{-}, \\
T_{(-1 / 2)(1 / 2)}^{L} & =T_{(1 / 2)(-1 / 2)}^{R}=-\sqrt{2} q_{+} .
\end{aligned}
$$

(iii) The propagation of $K_{J}^{*}$ is parameterized by a Breit-Wigner formula while the $K_{J}^{*} \rightarrow K \pi$ decay is described by the spherical harmonic functions: $Y_{J}^{i}\left(\theta_{K}, 0\right)$, with $i=0$ for $K_{0}^{*}$ and $i=0, \pm 1$ for $K_{J}^{*}$. It should be pointed out that the dependence of the coupling between a virtual $K_{J}^{*}$ and the $K \pi$ state on $m_{K \pi}^{2}$ is neglected. Since there is no singularity in the coupling, it can be expanded in terms of $m_{K \pi}^{2}-m_{K_{J}^{*}}^{2}$ around the resonance region, which is also guaranteed by the Breit-Wigner propagation. For definiteness, we list the explicit forms of the spherical harmonic functions used in this work

$$
\begin{aligned}
& Y_{0}^{0}\left(\theta_{K}, \phi\right)=\frac{1}{\sqrt{4 \pi}}, \quad Y_{1}^{0}\left(\theta_{K}, \phi\right)=\sqrt{\frac{3}{4 \pi}} \cos \theta_{K}, \quad Y_{1}^{ \pm 1}\left(\theta_{K}, \phi\right)=\mp \sqrt{\frac{3}{8 \pi}} \sin \theta_{K}, \\
& Y_{2}^{0}\left(\theta_{K}, \phi\right)=\sqrt{\frac{5}{16 \pi}}\left(3 \cos ^{2} \theta_{K}-1\right), \quad Y_{2}^{ \pm 1}\left(\theta_{K}, \phi\right)=\mp \sqrt{\frac{15}{32 \pi}} \sin \left(2 \theta_{K}\right), \quad Y_{3}^{0}\left(\theta_{K}, \phi\right)=\sqrt{\frac{7}{16 \pi}}\left(5 \cos ^{3} \theta_{K}-3 \cos \theta_{K}\right), \\
& Y_{3}^{ \pm 1}\left(\theta_{K}, \phi\right)=\mp \sqrt{\frac{21}{64 \pi}} \sin \theta_{K}\left(5 \cos ^{2} \theta_{K}-1\right), \quad Y_{4}^{0}\left(\theta_{K}, \phi\right)=\frac{3}{16 \sqrt{\pi}}\left(35 \cos ^{4} \theta_{K}-30 \cos ^{2} \theta_{K}+3\right), \\
& Y_{4}^{ \pm 1}\left(\theta_{K}, \phi\right)=\mp \frac{3 \sqrt{5}}{8 \sqrt{\pi}} \sin \theta_{K}\left(7 \cos ^{3} \theta_{K}-3 \cos \theta_{K}\right) .
\end{aligned}
$$

[1] B. Aubert et al. (BABAR Collaboration), Phys. Rev. Lett. 102, 091803 (2009).

[2] J. T. Wei et al. (BELLE Collaboration), Phys. Rev. Lett. 103, 171801 (2009).

[3] T. Aaltonen et al. (CDF Collaboration), Phys. Rev. D 79, 011104 (2009).

[4] The LHCb Collaboration, LHCb-CONF-2011-038.

[5] C. Bobeth, G. Hiller, and D. van Dyk, J. High Energy Phys. 07 (2011) 067; See also A. Ali, P. Ball, L. T. Handoko et al., Phys. Rev. D 61, 074024 (2000); F. Kruger and J. Matias, Phys. Rev. D 71, 094009 (2005); U. Egede, T. Hurth, J. Matias, M. Ramon, and W. Reece, J. High Energy Phys. 11 (2008) 032; W. Altmannshofer et al., J. High Energy Phys. 01 (2009) 019; and many references therein.

[6] R. -H. Li, C. -D. Lu, and W. Wang, Phys. Rev. D 83, 034034 (2011).

[7] W. Wang, Phys. Rev. D 83, 014008 (2011).

[8] S. Rai Choudhury, A. S. Cornell, G. C. Joshi, and B. H. J. McKellar, Phys. Rev. D 74, 054031 (2006).

[9] S. R. Choudhury, A. S. Cornell, and N. Gaur, Phys. Rev. D 81, 094018 (2010).

[10] H. Hatanaka and K. C. Yang, Phys. Rev. D 79, 114008 (2009); Eur. Phys. J. C 67, 149 (2010).

[11] N. Katirci and K. Azizi, J. High Energy Phys. 07 (2011) 043.

[12] M. Junaid and M. J. Aslam, arXiv:1103.3934.
[13] T. M. Aliev and M. Savci, Phys. Rev. D 85, 015007 (2012).

[14] K. Nakamura et al. (Particle Data Group), J. Phys. G 37, 075021 (2010).

[15] G. Buchalla, A. J. Buras, and M.E. Lautenbacher, Rev. Mod. Phys. 68, 1125 (1996).

[16] A. J. Buras and M. Munz, Phys. Rev. D 52, 186 (1995).

[17] K. -C. Yang, Phys. Lett. B 695, 444 (2011).

[18] M. J. Dugan and B. Grinstein, Phys. Lett. B 255, 583 (1991).

[19] J. Charles, A. Le Yaouanc, L. Oliver, O. Pene, and J. C. Raynal, Phys. Rev. D 60, 014001 (1999).

[20] M. Wirbel, B. Stech, and M. Bauer, Z. Phys. C 29, 637 (1985).

[21] D. Scora and N. Isgur, Phys. Rev. D 52, 2783 (1995).

[22] H. -Y. Cheng and K. -C. Yang, Phys. Rev. D 83, 034001 (2011).

[23] H. -Y. Cheng and C. -K. Chua, Phys. Rev. D 81, 114006 (2010); 82, 059904 (2010).

[24] H. Y. Cheng, Y. Koike, and K. C. Yang, Phys. Rev. D 82, 054019 (2010).

[25] C. W. Bauer, S. Fleming, D. Pirjol, and I. W. Stewart, Phys. Rev. D 63, 114020 (2001).

[26] C. W. Bauer, S. Fleming, and M. E. Luke, Phys. Rev. D 63, 014006 (2000).

[27] C. W. Bauer, D. Pirjol, and I. W. Stewart, Phys. Rev. Lett. 87, 201806 (2001). 
[28] C. W. Bauer, D. Pirjol, and I. W. Stewart, Phys. Rev. D 65, 054022 (2002).

[29] C. W. Bauer, D. Pirjol, and I. W. Stewart, Phys. Rev. D 67, 071502 (2003).

[30] M. Beneke and D. Yang, Nucl. Phys. B 736, 34 (2006).

[31] F. De Fazio, T. Feldmann, and T. Hurth, Nucl. Phys. B733, 1 (2006); B800, 405 (2008).

[32] C. W. Bauer, D. Pirjol, I.Z. Rothstein, and I. W. Stewart, Phys. Rev. D 70, 054015 (2004).

[33] A. Jain, I.Z. Rothstein, and I. W. Stewart, arXiv:0706.3399.

[34] Y. Y. Keum, H. n. Li, and A. I. Sanda, Phys. Lett. B 504, 6 (2001); Phys. Rev. D 63, 054008 (2001); C. D. Lu, K.
Ukai, and M.Z. Yang, Phys. Rev. D 63, 074009 (2001); C. D. Lu and M. Z. Yang, Eur. Phys. J. C 23, 275 (2002).

[35] R. -H. Li, C. -D. Lu, W. Wang, and X. -X. Wang, Phys. Rev. D 79, 014013 (2009); W. Wang, Y. -L. Shen, Y. Li, and C. -D. Lu, Phys. Rev. D 74, 114010 (2006); Y. -L. Shen, W. Wang, J. Zhu, and C. -D. Lu, Eur. Phys. J. C 50, 877 (2007); C. s. Kim, Y. Li, and W. Wang, Phys. Rev. D 81, 074014 (2010).

[36] P. Colangelo, F. De Fazio, and W. Wang, Phys. Rev. D 81, 074001 (2010); 83, 094027 (2011); W. Wang, AIP Conf. Proc. No. 1317 (AIP, New York, 2011).

[37] R. Aaij et al. (LHCb Collaboration), arXiv:1112.4695, and also LHCb-CONF-2011-035. 